\title{
Mechanisms of epigenetic inheritance in children following exposure to abuse
}

\author{
Elissavet Damaskopoulou ${ }^{\bowtie}$, George P. Chrousos² ${ }^{2}$ Elias Eliopoulos ${ }^{3}$, Dimitrios Vlachakis ${ }^{3,4,5}$ \\ ${ }^{1}$ Agricultural University of Athens, Athens, Greece \\ 2 University Research Institute of Maternal and Child Health \& Precision Medicine, and UNESCO Chair on Adolescent Health Care, \\ National and Kapodistrian University of Athens, Aghia Sophia Children's Hospital, Athens, Greece \\ ${ }^{3}$ Laboratory of Genetics, Department of Biotechnology, School of Applied Biology and Biotechnology, Agricultural University of \\ Athens, Athens, Greece \\ ${ }^{4}$ Laboratory of Molecular Endocrinology, Center of Clinical, Experimental Surgery and Translational Research, Biomedical Research \\ Foundation of the Academy of Athens, Athens, Greece \\ ${ }^{5}$ School of Informatics, Faculty of Natural \& Mathematical Sciences, King's College London, London, United Kingdom \\ Competing interests: ED none; GPC none; EE none; DV none
}

Child abuse refers to any kind of bad treatment of a minor under 18, of a physical and/or emotional nature, sexual abuse, neglect and negligent conduct and exposure to danger.

Abuse, in any form, is known to have devastating effects on the later course of children's lives. What is not known yet, is whether the genes expressions of abused children raised in an institution is affected by the family and then the institutional environment in which they live the first years of their lives. In recent years, studies have shown that adults who have been exposed in their childhood to neglect, abuse and stress due to adverse environmental conditions, are more likely to develop chronic biological (organic) and psychological diseases such as depression, hypertension, diabetes, various cardiorespiratory and autoimmune diseases and this now seem to have a biological background. Similar studies have shown that as children grow older, they experience a variety of emotional, social and behavioural problems, including hyperactivity, learning difficulties and stress. DNA methylation is one of the mechanisms through which the epigenetic process occurs and the differentiation of DNA expression in these individuals.

Most studies in humans and animals have focused on epigenetic variations in genes called adversity genes. The most common of these is the glucocorticoid receptor (GR) gene, also known as NR3C1, which shows a differentiation in its expression. Other genes that studies have shown to be related to child abuse are FOXP1 and FOXP2 and FKBP5 and SLC6A4. The genetic and epigenetic interplay between the aforementioned genes is studied and presented herein in an effort to elucidate their role in the heritability of child abuse. 